\title{
SCREENING OF COSMOLOGICAL CONSTANT IN NON-LOCAL COSMOLOGY
}

\author{
$\operatorname{AUTHOR}(\mathrm{S})$ :
}

ZHANG, YING-LI; SASAKI, MISAO

\section{CITATION:}

ZHANG, YING-LI ...[et al]. SCREENING OF COSMOLOGICAL CONSTANT IN NON-LOCAL COSMOLOGY. International Journal of Modern Physics D 2012, 21(01): 1250006.

\section{ISSUE DATE:}

2012-01

URL:

http://hdl.handle.net/2433/154864

\section{RIGHT:}

(c) 2012 World Scientific Publishing Co.; This is not the published version. Please cite only the published version.; この論文は出版社版で ありません。引用の際には出版社版をご確認ご利用ください。 


\title{
Screening of cosmological constant in non-local cosmology
}

\author{
Ying-li Zhang* and Misao Sasaki ${ }^{\dagger}$ \\ Yukawa Institute for Theoretical Physics, Kyoto University, Kyoto 606-8502, Japan
}

(Dated: December 30, 2011)

\begin{abstract}
We consider a model of non-local gravity with a large bare cosmological constant, $\Lambda$, and study its cosmological solutions. The model is characterized by a function $f(\psi)=f_{0} e^{\alpha \psi}$ where $\psi=\square^{-1} R$ and $\alpha$ is a real dimensionless parameter. In the absence of matter, we find an expanding universe solution $a \propto t^{n}$ with $n<1$, that is, a universe with decelerated expansion without any fine-tuning of the parameter. Thus the effect of the cosmological constant is effectively shielded in this solution. It has been known that solutions in non-local gravity often suffer from the existence of ghost modes. In the present case we find the solution is ghost-free if $\alpha>\alpha_{c r} \approx 0.17$. This is quite a weak condition. We argue that the solution is stable against the inclusion of matter fields. Thus our solution opens up new possibilities for solution to the cosmological constant problem.
\end{abstract}

\section{INTRODUCTION}

After the discovery of the accelerated expansion of the current universe, there have been a lot of discussions about which side of the Einstein equation should be modified, either by adding an extremely small cosmological constant or something similar to the matter side of the equation or by modifying the gravity side of the equation. In particular, inspired by string theory which lives in higher dimensions, modified gravity theories have been attracting much attention in recent years. One of such is non-local gravity. Its cosmological effect was studied in [1], and various aspects of it have been studied [2].

Here we are interested in a different aspect of non-local gravity. By studying phenomenological modifications of the Einstein equation, it was suggested that non-local gravity may solve the cosmological constant problem [3]. Inspired by this work, an explicit mechanism to screen the cosmological constant in non-local gravity was discussed in detail [4]. Unfortunately, however, similar to the situation of many higher derivative theories [5], non-local gravity often suffers from the existence of a mode with the incorrect sign of the kinetic term, that is, a ghost. There has been some attempt to remedy this defect, for example, by adding an $F(R)$ term [ [6], but it has not been successful so far. However, it should be noted that the analysis was done only for modes around either flat or de Sitter (constant curvature) spacetime solutions.

In this paper, we consider a simple one-parameter family of non-local gravity models with cosmological constant $\Lambda$ and study their cosmological solutions. The models are characterized by a function $f(\psi)=f_{0} e^{\alpha \psi}$ where $\psi \equiv \square^{-1} R$, with $\alpha$ being the real, dimensionless parameter: See Eq. (1) below.

We assume the universe is spatially flat, and consider the case where there is no matter contribution. Very interestingly, we find a power-law solution $a \propto t^{n}$, where $a$ is the cosmic scale factor, with $n<1$. This implies that the effect of the cosmological constant is completely shielded to render the expansion of the universe decelerated. To be specific, as $\alpha$ varies from $-\infty$ to $+\infty, n$ increases monotonically from 0 to $1 / 2$. Thus the universe behaves like a radiation-dominated universe for $\alpha \gg 1$. Then we examine if the solution is free from a ghost. To our happy surprise, it is found that for a very wide range of the parameter $\alpha$, namely for $\alpha>\alpha_{c r} \approx 0.17$, the solution is found to be ghost-free. For this range of $\alpha$, we find $n>n_{c r} \approx 0.35$.

The paper is organized as follows. In Section 2 , we present the action for a general class of non-local gravity without specifying the form of the function $f(\psi)$, and derive the equations of motion for spatially flat cosmology. Then we derive the ghost-free condition of the model by making a conformal transformation from the original (Jordan) frame to the Einstein frame. In Section 3, we specialize the theory to a model characterized by the $f(\psi)=f_{0} e^{\alpha \psi}$ and derive its cosmological solutions. We then examine the ghost-free condition. Section 4 is denoted to conclusion.

\footnotetext{
* E-mail address: yingli@yukawa.kyoto-u.ac.jp

$\dagger$ E-mail address: misao@yukawa.kyoto-u.ac.jp
} 


\section{NON-LOCAL GRAVITY AND GHOST-FREE CONDITION}

\section{A. Action and equations of motion}

We consider a class of non-local gravity whose action is given by

$$
S=\int d^{4} x \sqrt{-g}\left\{\frac{1}{2 \kappa^{2}}\left[R\left(1+f\left(\square^{-1} R\right)\right)-2 \Lambda\right]+\mathcal{L}_{\text {matter }}(Q ; g)\right\},
$$

where $\kappa^{2}=8 \pi G, f$ is a function that characterizes the nature of non-locality with $\square^{-1}$ being the inverse of the d'Alembertian operator, $\Lambda$ is a (bare) cosmological constant and $Q$ stands for matter fields. For definiteness, we assume matter is coupled minimally to gravity. Therefore, the above may be regarded as an action in the Jordan frame.

In this simple class of non-local gravity, we may rewrite the action into a local form by introducing two scalar fields $\psi$ and $\xi$ as

$$
\begin{aligned}
S & =\int d^{4} x \sqrt{-g}\left[\frac{1}{2 \kappa^{2}}\{R(1+f(\psi))-\xi(\square \psi-R)-2 \Lambda\}+\mathcal{L}_{\text {matter }}\right] \\
& =\int d^{4} \sqrt{-g}\left[\frac{1}{2 \kappa^{2}}\left\{R(1+f(\psi)+\xi)+g^{\mu \nu} \partial_{\mu} \xi \partial_{\nu} \psi-2 \Lambda\right\}+\mathcal{L}_{\text {matter }}\right] .
\end{aligned}
$$

By varying the action with respect to $g_{\mu \nu}, \xi$ and $\psi$, respectively, one obtains the field equations as

$$
\begin{aligned}
0= & \frac{1}{2} g_{\mu \nu}\left\{R(1+f(\psi)+\xi)+g^{\alpha \beta}\left(\partial_{\alpha} \xi \partial_{\beta} \psi\right)-2 \Lambda\right\}-R_{\mu \nu}(1+f(\psi)+\xi) \\
& -\frac{1}{2}\left(\partial_{\mu} \xi \partial_{\nu} \psi+\partial_{\mu} \psi \partial_{\nu} \xi\right)-\left(g_{\mu \nu} \square-\nabla_{\mu} \nabla_{\nu}\right)(f(\psi)+\xi)+\kappa^{2} T_{\mu \nu}, \\
0= & \square \psi-R \\
0= & \square \xi-f^{\prime}(\psi) R
\end{aligned}
$$

where $f^{\prime}(\psi) \equiv d f / d \psi$.

For this class of models, we consider their cosmological solutions. We assume a spatially flat FLRW universe with the metric,

$$
d s^{2}=-d t^{2}+a^{2}(t) \delta_{i j} d x^{i} d x^{j} .
$$

With this assumption, Eqs. (3)-(5) reduce to

$$
\begin{aligned}
0 & =-3 H^{2}(1+f(\psi)+\xi)-\frac{1}{2} \dot{\xi} \dot{\psi}-3 H\left(f^{\prime}(\psi) \dot{\psi}+\dot{\xi}\right)+\Lambda+\kappa^{2} \rho, \\
0 & =\left(2 \dot{H}+3 H^{2}\right)(1+f(\psi)+\xi)-\frac{1}{2} \dot{\xi} \dot{\psi}+\left(\frac{d^{2}}{d t^{2}}+2 H \frac{d}{d t}\right)(f(\psi)+\xi)-\Lambda+\kappa^{2} P, \\
0 & =\ddot{\psi}+3 H \dot{\psi}+6 \dot{H}+12 H^{2}, \\
0 & =\ddot{\xi}+3 H \dot{\xi}+\left(6 \dot{H}+12 H^{2}\right) f^{\prime}(\psi),
\end{aligned}
$$

where a dot denotes the time derivative ${ }^{\circ}=d / d t, H=\dot{a} / a$, and $\rho=-T_{0}^{0}$ and $P=T_{i}^{i} / 3$ are the energy density and pressure of the matter fields, respectively.

\section{B. Ghost-free condition}

One may be able to find various interesting solutions of the theory given by the action (11) or (2), they may not be physically relevant if they contain a ghost mode, that is, a mode with a wrong sign of the kinetic term. Such a mode may be harmless at classical level, but it would lead to a disastrous consequence in general as soon as we quantize it. Hence it is better to avoid the existence of a ghost. The ghost-free condition for the theory (2) was discussed in [4]. Here for completeness, let us briefly summarize the result. 
To examine if the theory contains a ghost or not, it is convenient to make a conformal transformation of the metric to bring the action into the one in the Einstein frame, namely the conformal frame in which the gravitational part of the action (2) becomes purely Einstein.

For this purpose, in this subsection (and only in this subsection), we denote the metric in the Jordan frame by $\tilde{g}_{\mu \nu}$, and consider a conformal transformation,

$$
\tilde{g}_{\mu \nu}=\Omega^{2} g_{\mu \nu}
$$

Then we have

$$
\tilde{R}=\Omega^{-2}\left[R-6\left(\square \log \Omega+g^{\mu \nu} \nabla_{\mu} \log \Omega \nabla_{\nu} \log \Omega\right)\right] .
$$

Therefore we find that if we identify the conformal factor as

$$
\Omega^{-2}=1+f(\psi)+\xi,
$$

the gravitational part of the action becomes Einsteinian.

The action in the Einstein frame is given by

$$
S=\int d^{4} x \sqrt{-g}\left\{\frac{1}{2 \kappa^{2}}\left[R-6\left(\square \log \Omega+g^{\mu \nu} \nabla_{\mu} \log \Omega \nabla_{\nu} \log \Omega\right)+\Omega^{2} g^{\mu \nu} \nabla_{\mu} \xi \nabla_{\nu} \psi-2 \Omega^{4} \Lambda\right]+\Omega^{4} \mathcal{L}_{\text {matter }}\left(Q ; \Omega^{2} g\right)\right\}
$$

Introducing a new field $\phi$ by

$$
\phi \equiv \log \Omega=-\frac{1}{2} \log (1+f(\psi)+\xi),
$$

in place of $\xi$, the above may be written as

$$
S=\int d^{4} x \sqrt{-g}\left\{\frac{1}{2 \kappa^{2}}\left(R-6 \nabla^{\mu} \phi \nabla_{\mu} \phi-2 \nabla^{\mu} \phi \nabla_{\mu} \psi-e^{2 \phi} f^{\prime}(\psi) \nabla^{\mu} \psi \nabla_{\mu} \psi-2 e^{4 \phi} \Lambda\right)+e^{4 \phi} \mathcal{L}_{\text {matter }}\left(Q ; e^{2 \phi} g\right)\right\} .
$$

It is now easy to derive the ghost-free condition. Since there are only two scalar fields, the condition for the absence of a ghost is that the trace and the determinant of the kinetic term matrix are both positive. In the present case, it is readily seen that only the positivity of the determinant is sufficient, that is,

$$
\operatorname{det}\left|\begin{array}{cc}
6 & 1 \\
1 & e^{2 \phi} f^{\prime}(\psi)
\end{array}\right|>0
$$

In terms of the original fields, this condition is expressed as

$$
f^{\prime}(\psi)>\frac{1}{6}(1+f(\psi)+\xi)>0,
$$

where $1+f(\psi)+\xi>0$ is a necessary condition from Eq. (15). Later the above condition is used to examine if our cosmological solutions are free from ghosts or not.

\section{COSMOLOGICAL SOLUTIONS}

As a class of simple non-local gravity models, we consider the case when $f(\psi)$ is given by an exponential function,

$$
f(\psi)=f_{0} e^{\alpha \psi} .
$$

For this class of models, we look for cosmological solutions with the assumption that the scale factor is a power-law function of time $t, a \propto t^{n}$. We also assume the absence of matter fields, $\rho=P=0$. 


\section{A. cosmological solutions}

Under the assumption that $a \propto t^{n}$, we solve the system of equations (7) - (10). First we solve Eq. (9). Inserting $H=n / t$ to it, we find the solution for $\psi(t)$ as

$$
\psi(t)=\psi_{1} t^{1-3 n}-\frac{6 n(2 n-1)}{3 n-1} \ln t / t_{0},
$$

where $\psi_{1}$ and $t_{0}$ are two integral constants. For simplicity, we set $\psi_{1}=0$. It may be worth mentioning that $\psi$ is the variable that signifies the nonlocality of our theory, $\psi=\square^{-1} R$. The above choice of the integration constant corresponds to the (regularized) retarded integral of the inverse d'Alembertian operator. Then the function $f(\psi(t))$ takes the form,

$$
f=f_{0}\left(\frac{t}{t_{0}}\right)^{m} ; \quad m \equiv-\alpha \frac{6 n(2 n-1)}{3 n-1} .
$$

Inserting the above into Eq. (10), one obtains the solution for $\xi(t)$

$$
\xi(t)=\xi_{0}+\xi_{1} t^{1-3 n}-\frac{6 n(2 n-1)}{m(3 n+m-1)} \alpha f_{0}\left(\frac{t}{t_{0}}\right)^{m},
$$

where $\xi_{0}$ and $\xi_{1}$ are two integral constants. Again for simplicity, we set $\xi_{1}=0$ in the following. Inserting these solutions into Eq. (7), we find for consistency,

$$
\xi_{0}=-1, \quad m=2, \quad t_{0}^{2}=\frac{6 n(n+1)}{\Lambda} f_{0} .
$$

From the viewpoint of Eq. (10), the above solution corresponds to the retarded integral of the inverse d'Alembertian operator plus a specific value of the constant homogeneous solution $\xi_{0}=-1$. In particular the second equation determines the power-law index $n$,

$$
6 \alpha n^{2}+3(1-\alpha) n-1=0 .
$$

This equation has two real solutions, which are given by

$$
\begin{aligned}
& n_{1}=\frac{-3+3 \alpha+\sqrt{3\left(3 \alpha^{2}+2 \alpha+3\right)}}{12 \alpha}, \\
& n_{2}=\frac{-3+3 \alpha-\sqrt{3\left(3 \alpha^{2}+2 \alpha+3\right)}}{12 \alpha} .
\end{aligned}
$$

We plot them in Fig. 1 as a function of $\alpha$. From this figure, we see that an expanding solution is given by $n=n_{1}$ for any $\alpha$ and by $n=n_{2}$ for $\alpha<0$. In both cases, $n$ approaches $1 / 2$ for $|\alpha| \gg 1$, that is, the evolution of the universe looks like a radiation-dominated one. We note that from Eqs. (23) and (24), the solution for $\psi$ can be rewritten in the form,

$$
\psi(t)=\frac{1}{\alpha} \ln \left[\frac{\Lambda t^{2}}{6 f_{0} n(1+n)}\right] .
$$

Now let us examine the ghost-free condition (18). Noting that $1+f+\xi$ is given by

$$
1+f+\xi=f\left(1-\alpha \frac{3 n(2 n-1)}{3 n+1}\right)=f \frac{6 n}{3 n+1},
$$

we have

$$
\frac{6 f^{\prime}}{1+f+\xi}=\alpha \frac{3 n+1}{n}=\alpha\left(3+\frac{1}{n}\right) .
$$

Then an analytical calculation shows that

$$
\begin{aligned}
& \left.\frac{6 f^{\prime}}{1+f+\xi}\right|_{n_{1}}>1 \Longrightarrow \alpha>\alpha_{c r} \equiv \frac{1}{15}\left(7-2^{\frac{1}{3}}-2^{\frac{5}{3}}\right) \approx 0.17 \\
& \left.\frac{6 f^{\prime}}{1+f+\xi}\right|_{n_{2}}<1 \quad \text { for } \forall \alpha \in \mathbf{R} .
\end{aligned}
$$




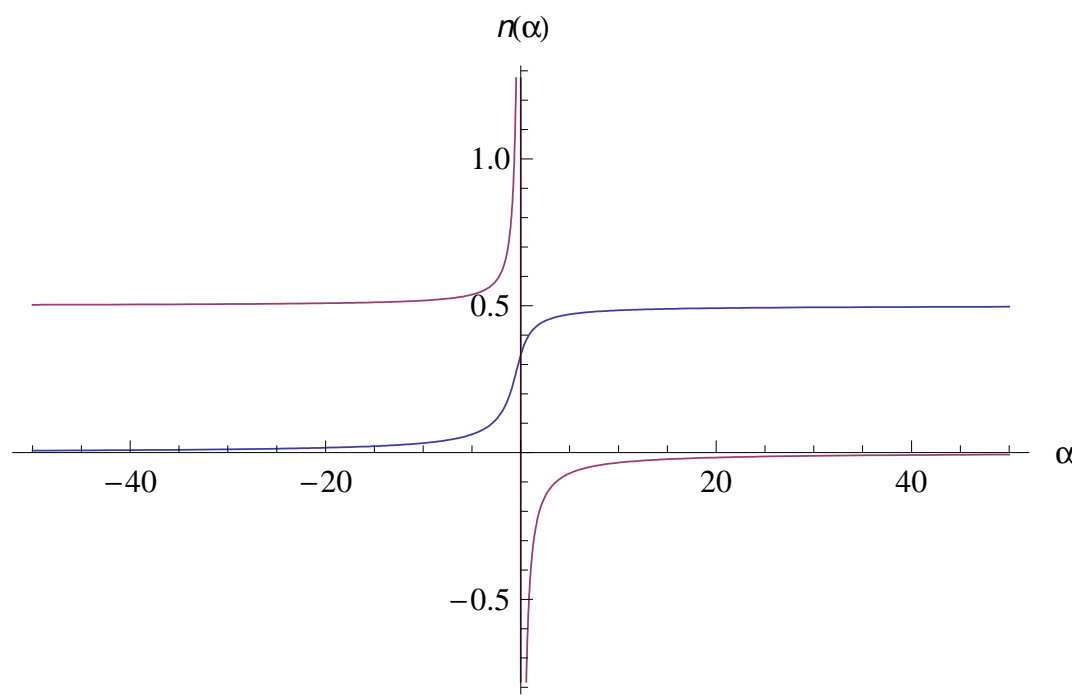

FIG. 1: Index $n$ as a function of parameter $\alpha$. Here the blue line (the one in the middle, which is regular at $\alpha=0$ ) denotes $n=n_{1}$ and the red line $n=n_{2}$.

So the ghost-free condition (18) implies that a ghost can be avoided for $n=n_{1}$ provided that the parameter $\alpha>$ $\alpha_{c r} \approx 0.17$, while the appearance of a ghost cannot be avoided at all for $n=n_{2}$.

In summary, we conclude that in our non-local gravity model with $f(\psi)=f_{0} e^{\alpha \psi}$, we have a cosmological solution,

$$
\psi(t)=\frac{1}{\alpha} \ln \left[\frac{\Lambda t^{2}}{6 f_{0} n(n+1)}\right], \quad \xi(t)=-1-\frac{(2 n-1) \alpha \Lambda t^{2}}{2(3 n+1)(n+1)},
$$

where

$$
n=n(\alpha) \equiv \frac{-3+3 \alpha+\sqrt{3\left(3 \alpha^{2}+2 \alpha+3\right)}}{12 \alpha},
$$

and the absence of a ghost is guaranteed provided that the parameter $\alpha$ satisfies

$$
\alpha>\alpha_{c r}=\frac{1}{15}\left(7-2^{\frac{1}{3}}-2^{\frac{5}{3}}\right) \approx 0.17 .
$$

Correspondingly, the power-law index $n$ varies in the range,

$$
1 / 2>n>n_{c r} \approx 0.35
$$

It should be noted that Eq. (18) implies $f^{\prime}(\psi)>0$, which reduces to the constraint on the parameter $f_{0}>0$ for a positive $\alpha$. Thus the solution we have obtained in the above is meaningful only for a positive cosmological constant $\Lambda$.

Finally, let us comment on the case when a matter field is present. If the coupling is as assumed in Eq. (11), that is, if matter is minimally coupled to gravity in the original conformal frame, then assuming the equation of state parameter $w=P / \rho$ is greater than minus one, $w>-1$, its effect will be always subdominant if it is so initially simply because its contribution to the energy density decreases faster than that of the cosmological constant. So our solution will be stable against the inclusion of matter fields.

\section{B. behavior in the Einstein frame}

In this subsection, we study the behavior of the solution (31) in the Einstein frame. The reason for doing this is the following. Although matter fields are assumed to be minimally coupled to gravity in the original frame as given in the action (11), this assumption has no relevance to our cosmological solution because we simply neglected their presence. In other words, we still have freedom in the choice of matter-gravity coupling. In particular, one may assume that a certain matter field is minimally coupled to gravity in the Einstein frame. Another reason is to confirm explicitly 
that the expansion is delecerated in the Einstein frame as well. ${ }^{1}$ Therefore it is useful to know the behavior of our cosmological solution in the Einstein frame.

In this subsection, we attach a subscript ${ }_{E}$ to quantities in the Einstein frame. Under the conformal conformal transformation $g_{\mu \nu}=\Omega^{2} g_{E, \mu \nu}$ the conformal factor is given by

$$
\Omega^{2}=\frac{1}{1+f+\xi}=\frac{(n+1)(3 n+1)}{\Lambda t^{2}},
$$

where $t$ is the time in the original frame.

The cosmic proper time $t_{E}$ in the Einstein frame is related to $t$ by $d t=\Omega d t_{E}$. Hence we obtain

$$
t_{E}=\int^{t} \frac{d t}{\Omega}=\frac{\sqrt{\Lambda} t^{2}}{2 \sqrt{(n+1)(3 n+1)}},
$$

where we have identified $t_{E}=0$ with $t=0$.

The scale factor in the Einstein frame is $a_{E}=\Omega a$. Hence the Hubble parameter in the Einstein frame can be calculated as

$$
H_{E}=\frac{1}{a_{E}} \frac{d a_{E}}{d t_{E}}=\frac{\Omega}{a} \frac{d}{d t_{E}}\left(\frac{a}{\Omega}\right)=\frac{\Omega^{2}}{a} \frac{d}{d t}\left(\frac{a}{\Omega}\right)=\frac{n+1}{2} \frac{1}{t_{E}},
$$

where we have used the fact $a \propto t^{n}$ and $\Omega \propto t^{-2}$ together with Eq. (36). This implies that the power-law index $n_{E}$ in the Einstein frame is given by $n_{E}=(n+1) / 2$, and takes the values in the range,

$$
0.75>n_{E}>n_{E, c r} \approx 0.675,
$$

for $\infty>\alpha>\alpha_{c r} \approx 0.17$.

Thus, from (36) and (37), we obtain the solutions for the scalar fields $\psi$ in the Einstein frame

$$
\begin{aligned}
& \psi\left(t_{E}\right)=\frac{1}{\alpha} \ln \left[\frac{1}{3 f_{0} n} \sqrt{\frac{\Lambda(3 n+1)}{n+1}} t_{E}\right], \\
& \xi\left(t_{E}\right)=-1-\alpha(2 n-1) \sqrt{\frac{\Lambda}{(3 n+1)(n+1)}} t_{E} .
\end{aligned}
$$

Again from Eq. (35), we note that the cosmological constant must be positive, $\Lambda>0$.

Before closing this section, an important comment is in order. As we demonstrated, the theory can be recasted in the form of a scalar-tensor theory or in the form of Einstein gravity plus two scalar fields. Therefore one might consider our theory to be simply a scalar-tensor theory that gives rise to a decaying cosmological constant. Technically this is true. But it should be emphasized that the form of the action for these scalar fields in either the original frame or the Einstein frame is completely fixed by the original form of non-local gravity given by Eq. (11): There is no freedom in maneuvering the form of the Lagrangian for these scalar fields. In other words, even if one were to regard this theory as a scalar-tenor theory with two non-minimally coupled scalar fields (in the original Jordan frame), it would be highly non-trivial to find a theory that would lead to a solution with a decaying cosmological constant which decays sufficiently fast in the original frame as well as in the Einstein frame.

\section{CONCLUSION AND DISCUSSION}

We have studied cosmological solutions in a simple class of non-local gravity with cosmological constant. The model is characterized by a function $f(\psi)=f_{0} e^{\alpha \psi}$ where $f_{0}>0$ and $\alpha$ is a real parameter, and $\psi$ is the inverse of the d'Alembertian acting on the scalar curvature, $\psi=\square^{-1} R$. In the absence of matter fields, we have found power-law solutions $a \propto t^{n}$ with $n<1$, that is, with decelerated expansion. We have found that for $\alpha>\alpha_{c r} \approx 0.17$, the solution is ghost-free. Thus without any fine-tuning, the solution successfully screens the effect of the cosmological constant that would have led to accelerated expansion.

\footnotetext{
${ }^{1}$ In fact, whether the expansion is delecerate or not does not depend on the choice of conformal frames, because it is directly related to the causal structure of the universe, which is conformal invariant by definition.
} 
We have assumed the absence of matter. If there is matter, we have to specify how the matter couples to gravity. If the matter couples minimally to gravity in the original (Jordan) frame, then it is expected that the presence of matter will not change the qualitative behavior of the solution in the sense that the solution we found will be an asymptotic, attractor solution of the system.

Our solution opens up new possibilities for the solution to the cosmological constant problem. It is known that the expansion of the present universe is accelerating. Therefore, in order to make our model more realistic, we must have matter which are not minimally coupled in the original frame, and which would lead to accelerated expansion at late times (but with a cosmological constant which is exponentially smaller than the original cosmological constant). Search for such a model is under way.

\section{Acknowledgments}

This work was supported in part by the Grant-in-Aid for the Global COE Program "The Next Generation of Physics, Spun from Universality and Emergence" from the Ministry of Education, Culture, Sports, Science and Technology (MEXT) of Japan, by JSPS Grant-in-Aid for Scientific Research (A) No. 21244033, and by JSPS Grant-in-Aid for Creative Scientific Research No. 19GS0219.

[1] S. Deser and R. P. Woodard, Phys. Rev. Lett. 99, 111301 (2007) [arXiv:0706.2151 [astro-ph]].

[2] L. Joukovskaya, Phys. Rev. D 76, 105007 (2007) [arXiv:0707.1545 [hep-th]];

S. Nojiri and S. D. Odintsov, Phys. Lett. B 659, 821 (2008) [arXiv:0708.0924 [hep-th]];

G. Calcagni, M. Montobbio and G. Nardelli, Phys. Lett. B 662, 285 (2008) [arXiv:0712.2237 [hep-th]];

T. Koivisto, Phys. Rev. D 77, 123513 (2008) [arXiv:0803.3399 [gr-qc]];

T. S. Koivisto, Phys. Rev. D 78, 123505 (2008) [arXiv:0807.3778 [gr-qc]];

T. Biswas, T. Koivisto and A. Mazumdar, JCAP 1011, 008 (2010) [arXiv:1005.0590 [hep-th]];

S. Capozziello, E. Elizalde, S. Nojiri and S. D. Odintsov, Phys. Lett. B 671, 193 (2009) [arXiv:0809.1535 [hep-th]];

N. A. Koshelev, Grav. Cosmol. 15, 220 (2009) [arXiv:0809.4927 [gr-qc]];

C. Deffayet and R. P. Woodard, JCAP 0908, 023 (2009) [arXiv:0904.0961 [gr-qc]];

S. Jhingan, S. Nojiri, S. D. Odintsov, M. Sami, I. Thongkool and S. Zerbini, Phys. Lett. B 663, 424 (2008) [arXiv:0803.2613 [hep-th]];

G. Cognola, E. Elizalde, S. Nojiri, S. D. Odintsov and S. Zerbini, Eur. Phys. J. C 64, 483 (2009) [arXiv:0905.0543 [gr-qc]];

H. Farajollashi and F. Milani, Int. J. Theor. Phys. 50, 1953 (2011) [arXiv:1103.3553 [gr-qc]];

J. Kluson, arXiv:hep-th/1105.6056;

D. J. Liu, B. Yang and X. H. Jin, arXiv:gr-qc/1108.2894.

[3] N. Arkani-Hamed, S. Dimopoulos, G. Dvali and G. Gabadadze, arXiv:hep-th/0209227.

[4] S. Nojiri, S. D. Odintsov, M. Sasaki and Y. l. Zhang, Phys. Lett. B 696, 278 (2011) [arXiv:1010.5375 [gr-qc]].

[5] J. Z. Simon, Phys. Rev. D 41, 3720 (1990).

[6] K. Bamba, S. Nojiri, S. D. Odintsov and M. Sasaki, arXiv:1104.2692 [gr-qc]. 